\title{
Modelo B-learning para el desarrollo del pensamiento crítico y creativo en estudiantes de una institución pública del Perú
}

\author{
Mg. Nikar Tatiana Aguirre Gonzales \\ agonzalesn@ucvvirtual.edu.pe \\ https://orcid.org/0000-0002-6084-6767
}

Mg. María Amparo de Dios Ruiz Sánchez

rsanchezma@ucvvirtual.edu.pe https://orcid.org/0000-0003-3061-3601

\author{
Dr. Aurelio Ruiz-Pérez \\ aruizpe@ucvvirtual.edu.pe \\ https://orcid.org/0000-0001-7684-3475 \\ Mg. Oscar Darwin Mendoza Cruzado \\ mcruzadood@ucvvirtual.edu.pe \\ https://orcid.org/0000-0002-4891-726X
}

Programa Académico de Doctorado en Educación

Escuela de postgrado Universidad César Vallejo

Chiclayo - Perú

\section{RESUMEN}

La presente investigación descriptiva-propositiva, está orientada a diseñar un modelo para fomentar el desarrollo del pensamiento crítico y creativo (PCC) a través de la enseñanza presencial y virtual en los estudiantes del VII ciclo de una institución pública. En esta investigación de enfoque cuantitativo se administraron dos cuestionarios, el primero desarrollado por los estudiantes, los cuales ayudaron a identificar el nivel en el que se encuentran con respecto al desarrollo del PCC, y el segundo aplicado a los docentes en relación al uso de las herramientas tecnológicas y su influencia en el desarrollo de los pensamientos en cuestión. Se identificó que la mayoría de los estudiantes participantes en la investigación se ubicaron en el nivel de proceso en ambos tipos de pensamiento, así como también en la variable B-learning. A raíz de estos resultados se elaboró la propuesta que fomentará en los estudiantes analizar, reflexionar y emitir opiniones, convirtiendo a 
las TIC en valiosos aliados que fortalezcan la enseñanza promoviendo el autoaprendizaje en ellos. Se difundió la propuesta planteada a través del fan page y Facebook de la institución, así como en el medio radial local para que la comunidad educativa se encuentre informada sobre el trabajo a realizar.

Palabras clave: pensamiento crítico; pensamiento creativo; herramientas tecnológicas; aprendizaje mixto. 


\title{
B-learning model for the development of critical and creative thinking in students of a public institution in Peru
}

\begin{abstract}
This descriptive-propositive research is aimed at designing a model to promote the development of critical and creative thinking (CCP) through face-to-face and virtual teaching in students of the VII cycle of a public institution. In this quantitative approach research, two questionnaires were administered, the first one developed by the students, which helped to identify the level at which they are with respect to the development of $\mathrm{CCP}$, and the second one applied to teachers in relation to the use of technological tools and their influence on the development of the thoughts in question. It was identified that most of the students participating in the research were at the process level in both types of thinking, as well as in the B-learning variable. As a result of these results, a proposal was developed to encourage students to analyze, reflect and issue opinions, turning TICs into valuable allies that strengthen teaching by promoting self-learning in them. The proposal was disseminated through the institution's fan page and Facebook, as well as in the local radio media so that the educational community is informed about the work to be done.
\end{abstract}

Keywords: critical thinking; creative thinking; technological tools; b-learning.

Artículo recibido: 15 enero 2022 Aceptado para publicación: 08 febrero 2022 Correspondencia: agonzalesn@ucvvirtual.edu.pe Conflictos de Interés: Ninguna que declarar 


\section{INTRODUCCIÓN}

En el actual contexto en el que vivimos, la pandemia ha modificado el estilo de vida de las familias de forma sustancial, provocando la clausura temporal de las instituciones educativas alrededor de todo el mundo y trasladando las sesiones de aprendizaje a los hogares, haciendo que la educación sea transmitida en forma virtual con el uso de las herramientas digitales. De ahí la necesidad de establecer estrategias donde los educandos desarrollen sus competencias, capacidades, habilidades y afronten los diversos escenarios que se les presenta en la vida. Para poder enfrentar estos retos es necesario implementar procedimientos que promuevan la aplicación de métodos y estrategias que potencien diversas destrezas como las del pensamiento crítico y creativo (PCC) orientado a la formación integral de los estudiantes.

UNESCO (2019) afirma que la educación se vio afectada por la falta de conectividad y los escasos recursos económicos que sufren las familias para obtener un dispositivo o tener acceso a internet, afectando principalmente a los sectores más pobres a nivel mundial. Ante ello se agregan las herramientas tecnológicas de comunicación donde la educación empieza a desarrollarse de forma abierta requiriendo de nuevos enfoques pedagógicos para desarrollar el PCC.

En Malasia como señala Firdausi et al. (2021) el desarrollo de las habilidades del PCC basado en proyectos educativos es relativamente bajo en los estudiantes del octavo grado, observando que desciende en las dimensiones fluidez y originalidad y asciende en flexibilidad y elaboración. Por lo tanto, se considera fundamental trabajar más con los estudiantes diversas estrategias, considerando el contexto para desarrollar el PCC de los mismos.

El Ministerio de investigación tecnológica y Educación Superior (MoRTHE) de Indonesia aplicó un proyecto piloto denominado SPADA (Sistem Pembelajaran Daring) que tuvo como finalidad elevar la calidad de la educación de los estudiantes a través de un aprendizaje mixto con el uso de las tecnologías. Este modelo elevó la efectividad de los aprendizajes tanto en forma sincrónica como asincrónica (Chaeruman et al., 2018). Por lo tanto, es fundamental la aplicación de modelos como el indicado considerando las características de los estudiantes y el contexto donde se ubican. Wechsler et al. (2018) expresan que en Brasil se vienen aplicando modelos alternativos, relacionados con la creatividad y el pensamiento crítico y la participación de los docentes con capacidades 
para desarrollar estas habilidades con los estudiantes de los diferentes niveles educativos interconectando con el rendimiento académico de las diferentes disciplinas de formación escolar.

Según el informe de la oficina de la medición de la calidad de los aprendizajes (UMC, 2020) los estudiantes del segundo grado de educación secundaria, obtuvieron un promedio de 567 puntos, nivel bajo, en lectura relacionado con los aspectos de analizar, identificar, inferir, que corresponden al pensamiento crítico. Niveles de esta naturaleza deben ser superados aplicando estrategias específicas y con ello superar las dificultades para interpretar, examinar, organizar, identificar, deducir, criticar sobre la información que leen.

La observación realizada a los estudiantes de educación secundaria de la institución educativa Cruz de Chalpón-Motupe, permitió determinar que tienen deficiencias en el desarrollo del pensamiento crítico y creativo teniendo como manifestaciones las dificultades en el razonamiento, poco analíticos, no reflexionan con fundamento, limitada participación en debates, con poca o casi nada argumentación para defender la postura o emitir una crítica, enjuiciamiento, así mismo, son poco originales en los trabajos escolares de las diferentes áreas curriculares.

Las causas de esta problemática son probablemente la limitada participación en debates a nivel de aula en las diferentes áreas curriculares, pocas oportunidades en debates públicos, en la realización de tareas escolares creativas, considerando su grado de estudio y el contexto, escasa participación en programas específicos como el B-learning con uso de las tecnologías de información y comunicación. Si la problemática continua, es decir no se llega a resolver, entonces los estudiantes serán entes pasivos, poco enjuiciadores o críticos con reducidas capacidades creativas en las diferentes áreas curriculares durante su formación en la educación secundaria, ubicándose en forma alejada en relación al desarrollo del pensamiento crítico y creativo, habilidades propias para los estudiantes del siglo XXI. Así mismo, va a resultar difícil contribuir con el logro de los objetivos planteados por la OCDE y la OSD relacionados con las competencias críticas y creativas a través de las actividades pedagógicas (OCDE, 2019).

El propósito de la investigación es que los estudiantes del VII ciclo de la institución educativa Cruz de Chalpón desarrollen y fortalezcan los niveles del PCC, a través de la consideración del modelo B-learning, fomentando el interés por investigar, indagar e 
identificar soluciones con facilidad durante el desarrollo educativo tanto virtual como presencial.

El problema quedó formulado de la siguiente manera: ¿Cómo el modelo B- learning es un componente que desarrolla el pensamiento crítico y creativo en los estudiantes del VII ciclo de la institución educativa Cruz de Chalpón de Motupe 2021? El objetivo general de la investigación es diseñar un modelo B-learning para el desarrollo del pensamiento crítico y creativo en los estudiantes del VII ciclo de la institución educativa Cruz de Chalpón, 2021. Los objetivos específicos son identificar el nivel de desarrollo del pensamiento crítico y creativo en los estudiantes mediante un cuestionario; elaborar el modelo B-learning para el desarrollo del pensamiento crítico y creativo en los estudiantes que participan en la investigación; validar el modelo B-learning para el desarrollo del pensamiento crítico y creativo en los estudiantes mediante juicio de expertos; difundir el modelo B-learning para el desarrollo del pensamiento crítico y creativo a través de medios de comunicación.

La investigación es relevante por estar orientada al desarrollo del PCC de los estudiantes de educación secundaria considerando el modelo B-learning, teniendo como aporte teórico a los resultados de la investigación presentados en tablas específicas y a la sistematización de información teórica relacionada con las variables de estudio. Metodológicamente la investigación tiene como aporte la estructuración de un modelo teórico centrado en B-learning y el desarrollo del PCC, considerando, las experiencias y actividades de aprendizaje.

En el aspecto social, el impacto está en el beneficio que tendrán los estudiantes desarrollando su pensamiento crítico y creativo a través del modelo B-learning en cuanto este sea aplicado con las exigencias del mismo, además, benefician también en forma indirecta a los padres de familia y a la comunidad donde se ubica la institución educativa considerada para la investigación.

La hipótesis quedó formulada con los siguientes términos, si se diseña un modelo Blearning para su aplicación en cuanto existan las condiciones, después de la pandemia covid-19, entonces se logra en forma óptima el desarrollo del pensamiento crítico y creativo en los estudiantes de la institución educativa Cruz de Chalpón-Motupe. 


\section{ESTRATEGIAS METODOLÓGÍCAS}

\subsection{Tipo y diseño de investigación}

La investigación es de tipo básica en su nivel propositivo, porque no se produjeron cambios en los estudiantes participantes, pero sí incrementa el conocimiento científico; como señala Tantaleán (2015) este tipo de investigación se orienta a diseñar una propuesta de cambio en una institución, debidamente argumentada generando beneficios a los integrantes en la investigación.

El diseño de investigación es de tipo descriptiva - propositiva toda vez que se hizo una propuesta relacionada con el modelo B-learning para el desarrollo del pensamiento crítico y creativo; de conformidad con Estela (2020) quien señala que el diseño descriptivo propositivo se representa en base al análisis que va desde la observación del objeto de estudio, dando lugar a un diagnóstico, la formulación del problema hasta el producto final que representa la solución a un problema determinado.

El diagrama del diseño es de creación propia, representado a continuación:

Figura 1 Diagrama de diseño

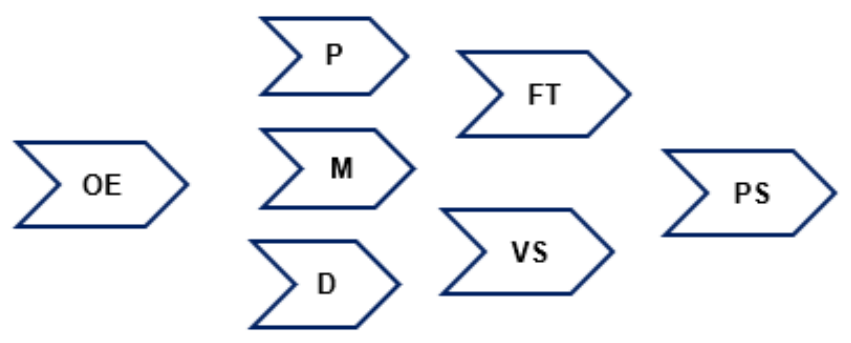

\section{Donde:}

OE : Objeto de estudio (pensamiento crítico y creativo)

P : Población

M : Muestra

D : Diagnóstico de los niveles del pensamiento crítico y creativo

FT : Análisis y fundamentación teórica.

VS : Variable solución (B-learning)

PS : Propuesta de solución.

Así mismo, es de enfoque metodológico cuantitativo, porque se trabajó con datos numéricos en forma estadística. La forma fiable para saber la realidad es por intermedio de la recolección y análisis de los datos cumpliendo con ciertas reglas lógicas (VegaMalagón, et al., 2014, p. 526). 


\subsection{Variables y Operacionalización}

\subsubsection{Variable dependiente: Pensamiento crítico y creativo}

El pensamiento crítico de acuerdo con Ennis (2018) es la mezcla de capacidades y habilidades que tiene el ser humano para tomar decisiones, de forma reflexiva y razonable. Involucra un análisis, que busca la veracidad por medio de criterios y evidencias, al igual que conseguir un juicio de valores.

Se define al pensamiento creativo como la producción de ideas o innovaciones a partir de algo existente o de algo que no existe dándole originalidad, donde se busca más allá de lo supuesto (García y Tigua, 2018).

Operacionalmente el pensamiento crítico fue definido como la capacidad que tiene cada ser humano para discernir, entender y tomar decisiones que puedan ayudar en la vida tanto personal como profesional. Y el pensamiento creativo es un proceso que permite elaborar ideas o proyectos innovadores que pueden partir de algo pequeño o de la nada.

\subsubsection{Variable independiente: B-learning}

Gil y Melo (2018) definen el B-learning como la enseñanza presencial y la virtual; generando una coherencia entre adaptabilidad y colaboración mutua, con ayuda de las TIC. El B-learning se definió operacionalmente como la fusión de la enseñanzaaprendizaje presencial y virtual tanto de manera sincrónica como asincrónica.

La operacionalización de las variables de estudio se presenta en el anexo 1, destacando las dimensiones e indicadores correspondientes.

\subsection{Población, muestra, muestreo}

Se contó con una población de 662 estudiantes del VII ciclo (tercer, cuarto y quinto grado) de la institución educativa "Cruz de Chalpón" de Motupe; se tuvo una población de 35 docentes según se evidencia en la tabla 1.

Tabla 1 Distribución del número de estudiantes, y docentes de la institución educativa Cruz de Chalpón-2021

\begin{tabular}{|l|c|c|c|}
\hline \multicolumn{1}{|c|}{ EII Ciclo } & varones & mujeres & total \\
\hline Tercer grado & 107 & 121 & 228 \\
\hline Cuarto grado & 97 & 111 & 208 \\
\hline Quinto grado & 89 & 102 & 191 \\
\hline Docentes & 13 & 22 & 35 \\
\hline TOTAL & 306 & 356 & 662 \\
\hline
\end{tabular}

Nota: Nómina de matrícula de los estudiantes de la I.E Cruz de Chalpón - Motupe 2021.Cuadro de asignación del personal 
Se determinó como muestra de estudio a 240 estudiantes y 35 docentes del VII ciclo de educación secundaria, para esto, se aplicó la fórmula del tamaño de muestra (anexo 4) con un margen de error de 5\% y un nivel de confianza de 95\%. La muestra es un subgrupo del universo de la población conformado por estudiantes que comparten características comunes (Hernández, Fernández y Baptista, 2014).

Los estudiantes participantes en la investigación fueron seleccionados utilizando la técnica del azar simple, para ello se dispuso de las nóminas de cada grado del VII ciclo, habiendo determinado contar con 80 estudiantes de cada grado, usando balotas e identificando en la nómina respectiva. Con respecto a los docentes participantes la muestra fue el $100 \%$ de docentes (35) que laboran con los estudiantes del VII ciclo. Esta técnica garantiza la participación de todos los individuos que componen la población con las mismas oportunidades de ser consideradas en la muestra (Otzen y Manterola, 2017).

\subsection{Técnicas e instrumentos de recolección de datos}

La encuesta se aplicó mediante dos cuestionarios administrado a los estudiantes y docentes de la institución educativa Cruz de Chalpón, en forma diversificada, con estructura especial y considerando la escala Likert, en relación al pensamiento crítico y creativo y al modelo B-learning, con herramientas tecnológicas considerando sesiones de aprendizaje presenciales y virtuales. Según explica Hernández (2012) la técnica de la encuesta se usa para recoger información de personas respecto a características, opiniones, creencias, con lo cual se obtiene y elabora datos de forma rápida y eficaz La validez de los instrumentos de la investigación estuvo a cargo de seis expertos cuyos resultados fueron procesados con la $\mathrm{V}$ de Aiken, obteniéndose un coeficiente igual a 0,9, valor significativo dando seguridad para su aplicación (procesamiento-anexo 6).

La confiabilidad se aplicó a través de dos pruebas piloto; una a 30 estudiantes y la otra a 15 docentes seleccionados de forma aleatoria de los diferentes grados y secciones del VII ciclo de la institución educativa Augusto B. Leguía (anexo 4), la cual la población tiene características similares a la institución donde se realizó el estudio, siendo procesados los resultados con el Alfa de Cronbach. La confiabilidad del Alfa de Cronbach se utiliza para evaluar la confiabilidad y esta debe ser cercana a uno para ser significativa (Hernández y Torres, 2018).

\subsection{Procedimientos}

Los procedimientos que se utilizaron en la presente investigación están relacionados con 
las coordinaciones con el personal directivo de la institución educativa, para obtener el permiso sobre la realización de la investigación, por lo tanto, incluye la aplicación de los instrumentos de investigación. Coordinaciones con los docentes, estudiantes y padres de familia para lograr su participación efectiva previo el consentimiento informado relacionado con las variables de estudio, aplicando el formulario Google mediante la aplicación del WhatsApp.

\subsection{Método de análisis de datos}

Los datos registrados posterior a la aplicación de los instrumentos de investigación, fueron procesados estadísticamente utilizando el programa Excel, así como el SPSS V26, determinando medidas de tendencia central y de dispersión, derivando tablas estadísticas en base a las cuales se hizo el análisis correspondiente.

\subsection{Aspectos Éticos}

La investigación se llevó a cabo en el marco de la ética de investigación, en base al código de ética de la Universidad César Vallejo (2020) que señala que se trabaja la confidencialidad al guardar reserva de los informantes y la libertad para desarrollar la investigación de manera libre e independiente además de garantizar el respecto a cada uno de los autores citándolos de forma correcta a través de la Norma APA en su 7ma edición.

\section{RESULTADOS}

En esta parte de la investigación se presentan los resultados de forma organizada y relacionada con los parámetros, métricas y criterios que permitieron aplicar los cuestionarios a los estudiantes y docentes.

Resultados de las variables pensamiento crítico - creativo y B-learning

Tabla 13 Comparación de las variables del pensamiento crítico - creativo y el modelo Blearning

\begin{tabular}{|l|c|c|c|c|c|c|}
\hline \multicolumn{1}{|c|}{ Niveles } & \multicolumn{2}{|c|}{ Pensamiento Crítico } & \multicolumn{2}{c|}{ Pensamiento Creativo } & \multicolumn{2}{c|}{ B-learning } \\
\hline & $\mathrm{F} 1$ & $\%$ & $\mathrm{~F} 1$ & $\%$ & $\mathrm{~F} 1$ & $\%$ \\
\hline En inicio & 1 & 0,4 & 1 & 0,4 & 1 & 2,9 \\
\hline En proceso & 222 & 92,5 & 215 & 89,6 & 31 & 88,6 \\
\hline Logro esperado & 15 & 6,3 & 20 & 8,3 & 2 & 5,7 \\
\hline Logro destacado & 2 & 0,8 & 4 & 1,7 & 1 & 2,9 \\
\hline Total & 240 & 100,0 & 240 & 100,0 & 35 & 100,0 \\
\hline
\end{tabular}

Nota: cuestionario aplicado a estudiantes 
En relación a la variable del pensamiento crítico, de 240 estudiantes encuestados, 222 que representan el $92,5 \%$ y son la gran mayoría de estudiantes encuestados, se encuentran en el nivel proceso, es decir que aún no han desarrollado totalmente sus habilidades en relación al pensamiento crítico, 15 de ellos que hacen un 6,3\% se encuentran en el nivel esperado, 2 participantes que equivale a un 0,8\% tienen desarrolladas las habilidades de la variable mencionada, y sólo 1 de ellos se encuentra en el nivel inicio.

Así mismo, referente al pensamiento creativo, 215 estudiantes que equivale a un 89,6\% y que es el nivel que tiene la mayor cantidad de participantes, se encuentran en el nivel proceso, es decir, aún tienen dificultades en desarrollar sus habilidades creativas, 20 estudiantes que hacen un $8,3 \%$ se encuentran en el nivel esperado, solo 4 participantes que equivale al $1,7 \%$ han desarrollado satisfactoriamente sus habilidades creativas mientras que 1 estudiante que equivale al $0,4 \%$ aún no desarrolla las habilidades anteriormente mencionadas.

Finalmente, referente a la variable B-learning, de 35 docentes encuestados, 31 de ellos que equivale al $88,6 \%$ que son la gran mayoría, consideran que los estudiantes se encuentran en el nivel en proceso, 2 de ellos que equivale el 5,7\% manifiestan que se encuentran en el nivel esperado, y solo se encuentra 1 docente tanto en el nivel inicio como el nivel destacado que equivale $2,9 \%$ cada uno.

\section{DISCUSIÓN}

Hoy en día, cuando hablamos de educación no podemos dejar de relacionarla con las herramientas tecnológicas, el uso de ellas ha contribuido en la evolución de la enseñanza, así como ha modificado el rol de los docentes en el aprendizaje de sus estudiantes, debido a que han dejado de ser no solo transmisores de conocimientos, sino que han aprendido a elaborar diferentes estrategias transformadoras con el uso de los entornos virtuales para conseguir que los estudiantes obtengan aprendizajes significativos que potenciarán su autoaprendizaje, a ser innovadores; a guiarlos para que construyan ellos mismos sus conocimientos, en tal sentido, los entornos virtuales se han convertido en parte fundamental de su existencia tanto en su vida familiar, social como educacional.

Los resultados derivados de la presente investigación se han discutido tomando en cuenta las bases teóricas científicas, objetivos e hipótesis planteados que demostraron cómo el uso de las herramientas tecnológicas va a desarrollar el pensamiento crítico y creativo (PCC) en los estudiantes. El PCC que han desarrollado los estudiantes a través de la 
enseñanza presencial con ayuda de las herramientas tecnológicas es una inquietud que se ha tomado en el objetivo general de esta investigación, que está centrado en diseñar un modelo B-learning para el desarrollo del PCC en la institución educativa Cruz de Chalpón, Motupe, 2021. Para responder a los objetivos planteados en esta investigación, primero se realizó dos pruebas pilotos a 30 estudiantes y 15 docentes respectivamente del VII ciclo que estudian y enseñan en la institución educativa Augusto Bernandino Leguía del distrito de Mochumi, quienes tienen características similares a los participantes de la institución educativa donde se realizó la investigación para comprobar la veracidad y eficacia del instrumento.

Una vez comprobada la confiabilidad y validez del instrumento, el siguiente paso fue convocar a una reunión vía zoom con las docentes del área elegida para coordinar como se aplicaría el cuestionario y el permiso a los padres a través de sus grupos de WhatsApp (previo permiso y autorización del líder pedagógico de la institución). El primer cuestionario se aplicó a una muestra de 240 estudiantes de tercero, cuarto y quinto grado de secundaria distribuidos proporcionalmente usando la técnica del azar simple con ayuda de las nóminas de matrícula 2021 de la institución. El segundo cuestionario se aplicó a los 35 docentes que enseñan en el VII ciclo, es decir el total de la población de docentes participaron como muestra de estudio, la cual garantizo las mismas posibilidades de participación a todos los individuos que forman parte de la población.

Las estrategias que se tomaron para la recolección de datos de los estudiantes se realizaron de 3 formas; la primera fue enviar el cuestionario a través de la aplicación Google Form, donde la mayoría de los estudiantes respondieron a esta primera estrategia; la segunda estrategia fue con capturas de pantalla para que marquen las respuestas y finalmente a través de llamadas telefónicas, lográndose el objetivo final a los 17 días de ser aplicado el cuestionario. El cuestionario aplicado a los estudiantes se basó en las dimensiones tanto del pensamiento crítico como del creativo donde se plantearon algunos objetivos específicos, que son abordados a continuación. El primer objetivo específico está orientado a identificar el nivel de desarrollo del PCC en los estudiantes. Con respecto al pensamiento crítico y creativo, Wechsler et al. (2018), manifiestan que tanto el pensamiento crítico como el creativo son diferentes, pero que ambas son habilidades que se encaminan a solucionar problemas que van apareciendo a lo largo de la vida, así como 
fomentar la creatividad y por consiguiente va a mejor el rendimiento académico de cada uno de los estudiantes.

En relación a la variable del pensamiento crítico, de 240 estudiantes encuestados, 222 estudiantes que son la gran mayoría de estudiantes encuestados, se encuentran en el nivel proceso, es decir que aún no han desarrollado totalmente sus habilidades en relación al pensamiento crítico, 15 de ellos se encuentran en el nivel esperado, 2 estudiantes tienen desarrolladas las habilidades de la variable mencionada, y solo uno de ellos se encuentra en el nivel inicio, Así mismo, referente al pensamiento creativo, 215 estudiantes se encuentran en el nivel proceso, es el nivel que tiene la mayor cantidad de participantes, 20 de ellos se encuentran en el nivel esperado, 4 estudiantes han desarrollado satisfactoriamente sus habilidades creativas mientras que 1 estudiante aún no desarrollan las habilidades anteriormente mencionadas.

Referente a la variable B-learning, de 35 docentes encuestados que enseñan el VII ciclo, 31 docentes que son la gran mayoría, consideran que los estudiantes se encuentran en el nivel en proceso, 2 docentes manifiestan que los estudiantes se ubican en el nivel esperado, y solo un docente considera que se encuentran en el nivel destacado y en el nivel inicio respectivamente.

Estos resultados de las variables tanto del pensamiento crítico como creativo guardan relación con el informe de la oficina de la medición de la calidad de los aprendizajes (UMC, 2020), que en el año 2019, los estudiantes del segundo grado de secundaria, se ubicaron en el primer nivel, es decir, que solo lograron aprendizajes muy básicos, el $17,7 \%$ de participantes se encontraron en el nivel previo al inicio; 42,2\% en inicio; $25,8 \%$ en proceso y $14,5 \%$ en satisfactorio. Por consiguiente, los estudiantes presentan grandes dificultades para interpretar, examinar, organizar, identificar, deducir, criticar sobre la información que leen.

Por esta razón, se determinó que es indispensable solucionar problemas del pensamiento crítico y creativo por medio de propuestas de modelos que promuevan una mejoría, en consecuencia, se ha propuesto un Modelo B-learning para el desarrollo del pensamiento crítico y creativo en estudiantes de la Institución Educativa “Cruz de Chalpón”-Motupe. Para la propuesta se tendrá como base al estudio realizado por Papert (1984), quien señala que el estudiante es el principal agente en el aprendizaje activo, el cual relaciona el nuevo conocimiento con el que ya había adquirido, donde el docente se convierte en facilitador 
del aprendizaje, cuya función es la de apoyar a cimentar plataformas para impulsar otros saberes, estimulando a los estudiantes en la adquisición de nuevos conocimientos, donde necesariamente tienen que estar involucrados tanto docentes como estudiantes.

También se consideró al estudio de Jerome Bruner (1960) que señala que los estudiantes deben de descubrir sus aprendizajes por medio de la curiosidad donde van a emplear la imaginación, así como intuición y creatividad para resolver problemas. La labor del docente en esta teoría es la de alcanzar los materiales apropiados para incentivar a los estudiantes.

La última teoría a considerar es la de Ausubel (1963) que implica insertar contenidos curriculares que posean sentido y lógica, para ello, es fundamental que los estudiantes tengan conocimientos previos en sus estructuras cognitivas para que el aprendizaje tenga sentido, quiere decir, que el estudiante cree y construya sus conocimientos para luego procesarlos y finalmente aplicarlos a la realidad. Por lo tanto, diremos que las tres teorías mencionadas ayudarán en la propuesta planteada en diseñar un modelo híbrido de enseñanza que ayudará a los estudiantes a descubrir, analizar, inferir para tener la capacidad de resolver problemas concretos utilizando el pensamiento crítico y creativo. Finalmente, los cuestionarios para evaluar el pensamiento crítico y creativo, como el del modelo B-learning para el desarrollo del pensamiento crítico y creativo en los estudiantes de la Institución Educativa "Cruz de Chalpón” de Motupe, fueron validados a criterio de seis expertos con Doctorado en Educación, quienes dieron su conformidad tanto en el diseño como en la aplicabilidad del mismo.

\section{CONCLUSIONES}

Después de aplicar los cuestionarios a estudiantes como docentes, se diseñó un modelo B-learning que ayudará a los estudiantes a un mejor manejo de las herramientas tecnológicas en sus actividades educativas para fomentar el desarrollo del pensamiento crítico y creativo.

En la investigación realizada se identificó los niveles de desarrollo del pensamiento crítico y creativo, resultando que la mayoría de los estudiantes del VII ciclo de la institución educativa se encuentran en el nivel proceso en ambas variables.

Identificado los niveles de desarrollo del PCC, se elaboró la propuesta denominada "Modelo B-learning para el desarrollo del pensamiento crítico y creativo", la cual ayudará a que los estudiantes se encuentren motivados para realizar sus trabajos pedagógicos a 
través de las herramientas tecnológicas con ayuda de los docentes, de esta manera, convertir a las Tics en aliados estratégicos que fortalezcan la enseñanza y promuevan el aprendizaje en los estudiantes.

La propuesta presentada fue validada por 5 expertos considerando el Coeficiente de validación de contenido (CVC), quienes dieron la aprobación para su aplicación.

La propuesta fue difundida a través del fan page y Facebook de la institución educativa “Cruz de Chalpón”, así como en el medio de comunicación radial local para que la comunidad educativa se encuentre informada sobre el trabajo que se realizará en dicha institución.

\section{PROPUESTA}

\section{Modelo B-learning para el desarrollo del pensamiento crítico y creativo en los estudiantes de la institución educativa Cruz de Chalpón-Motupe, 2022}

B-learning conocido en inglés como blended learning es un enfoque de aprendizaje que combina la enseñanza presencial dictada por un docente y la enseñanza de aprendizaje virtual. Para este tipo de trabajo los docentes formadores incorporan las herramientas tecnológicas para mejorar la experiencia de aprendizaje de los estudiantes, así como desarrollar el pensamiento crítico y creativo en cada sesión de clase. Esta nueva forma de compartir el aprendizaje aprovecha las virtudes de la educación presencial y de la modalidad en línea, enfatizando el uso de las TIC para permitir la mejora de la experiencia educativa tanto en docentes como en estudiantes.

El modelo en mención apareció como apoyo a la plana docente, ya que permite nuevos enfoques y modalidades para el trabajo tanto individual como grupal, así como fomentar juicios críticos en los estudiantes

Por lo tanto, la presente propuesta tiene como finalidad la integración de las herramientas digitales con la enseñanza presencial para desarrollar el pensamiento crítico y creativo en los estudiantes, con ello se busca poner en marcha las estrategias innovadoras relacionadas con las tecnologías educativas para que los estudiantes puedan potenciar su propio aprendizaje.

La implementación de la propuesta, tal como se detalla en el anexo 10, será organizado en 09 sesiones participativas de 03 horas cada una; las mismas que primero serán aplicadas a los docentes de la institución, lo cual fortalecerá el mejoramiento de las capacidades y actitudes de los estudiantes. De la misma manera, los estudiantes serán 
Modelo B-learning para el desarrollo del...

capacitados en horarios programados por grados y secciones para fortalecer sus conocimientos y el dominio de las tecnologías que ayudarán a mejorar el pensamiento crítico y creativo en ellos. 
Figura 2: Representación de la propuesta de investigación.

Modelo B-learning para el desarrollo del pensamiento crítico y creativo

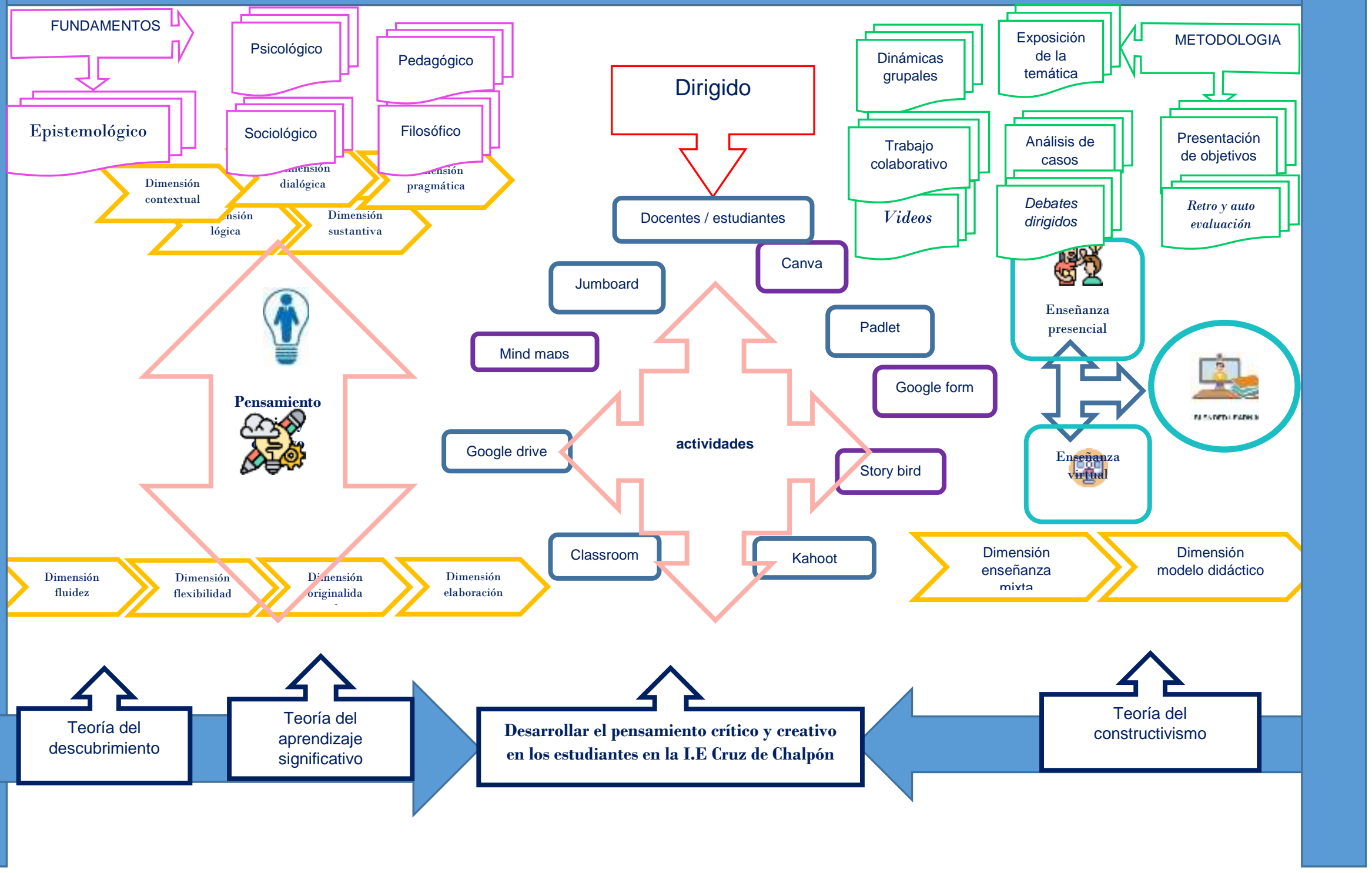

Ciencia Latina Revista Científica Multidisciplinar, Ciudad de México, México.

ISN 2707-2207 / ISSN 2707-2215 (en línea), enero-febrero, 2022, Volumen 6, Número 1. https://doi.org/10.37811/cl rcm.v6i1.1771 p 3843 


\section{REFERENCIAS BILIOGRAFICAS}

Alpysov, A., Kireyeva, A., Kadkalova, T., Dautova, Z., Popova, M., \& Zhubandykova, A. (2017). On the development of mathematical competencies of students in the construction and solution of complex inequalities. Revista Espacios, 38(50), 1-9. http://www.revistaespacios.com/a17v38n50/a17v38n50p31.pdf

Ausubel, D. P. (1963). The psychology of meaningful verbal learning.

Bruner, J. S., Goodnow, J. J., \& Austin, G. A. (2017). A study of thinking. Routledge.

Chaeruman, U. A., Wibawa, B., \& Syahrial, Z. (2018). Determining the appropriate blend of blended learning: A formative research in the context of SpadaIndonesia. American Journal of Educational Research, 6(3), 188-195. Psicogente, 13(24), 329-346. https://www.redalyc.org/pdf/4975/497552357008.pdf.

Ennis, R. H. (2018). Critical thinking across the curriculum: A vision. Topoi, 37(1), 165184.

Firdausi, Y., Sujadi, I., \& Nurhasanah, F. (2021). Students' Creative Thinking Process in Solving Ill-Structured Problem at Eight Grade Students with High Ability. Journal of Physics, 19(18), 1-7. https://iopscience.iop.org/article/10.1088/17426596/1918/4/042071/pdf

García Vallejo, S. S., \& Tigua Ávila, D. J. (2018). Recursos didácticos ecológicos en el desarrollo del pensamiento creativo del subnivel medio (Bachelor's thesis, Universidad de Guayaquil. Facultad de Filosofiía, Letras y Ciencias de la Educación.).

Gil Toledo, J. J., \& Melo Melo, C. M. (2018). El Blended learning; instrumento generador de formación de alto impacto.

Hernández, O. (2012). Estadística Elemental para Ciencias Sociales. (Tercera Edición). San José, Costa Rica: Editorial Universidad de Costa Rica.

Hernández Sampieri, R, Fernández, C \& Baptista, P. (2014). Metodología de la Investigación. (Quinta Edición). México D.F, México: McGraw-Hill.

Hernández-Sampieri, R., \& Torres, C. P. M. (2018). Metodología de la investigación (Vol. 4). México eD. F DF: McGraw-Hill Interamericana.

Ismajli, H., Bytyqi-Damoni, A., Shatri, K., \& Ozogul, G. (2020). Coaching teachers to integrate technology: The effects of technology integration on student performance and 
Meléndrez, K. F. A. (2019). Investigación, desarrollo e innovación en el ámbito educativo: aportaciones desde la psicología. Revista Electrónica de Psicología Iztacala [Internet], 22(2), 2187-2213.

OCDE. (2019). Estrategia de competencias de la OCDE 2019. competencias para construir un futuro mejor. In Fundación Santillana, 2019. para la edición en español. https://doi.org/10.1787/e3527cfb-es

Otzen, T., \& Manterola, C. (2017). Técnicas de Muestreo sobre una Población a Estudio. International journal of morphology, 35(1), 227-232.

Papert, S. (1984). Desafíos de la mente. Computadoras y educación. https://educavallologo.files.wordpress.com/2016/12/capitulo-7-desafio-a-la-mente seymour-papert.pd

R.C.U. N ${ }^{\circ}$ 0262-2020/UCV actualización del código de ética en investigación de la universidad Cesar Vallejo https://www.ucv.edu.pe/wpcontent/uploads/2020/11/RCUN\%C2\%B00262-2020-UCV-Aprueba-

Actualizaci\%C3\%B3n-del-C\%C3\%B3digo-\%C3\%89tica-en-

Investigaci\%C3\%B3n-1-1.pdf

Silva, J. (2017). Un modelo pedagógico virtual centrado en las E-actividades. Revista de Educación a Distancia (RED), (53)

Tantalean, R. M.T. (2015). El alcance de las investigaciones jurídicas. AVANCES, 10(11), 221-221.

UMC. (2020). ¿Qué aprendizajes logran nuestros estudiantes? Resultados de las evaluaciones nacionales de logros de aprendizaje 2019. Wechsler, S. M., Saiz, C., Rivas, S. F., Vendramini, C. M. M., Almeida, L. S., Mundim, M. C., \& Franco, A. (2018). Creative and critical thinking: Independent or overlapping components? Thinking Skills and Creativity, 27, 114-122. http://umc.minedu.gob.pe/wp-content/uploads/2020/06/Reporte-Nacional2019.pdf

UNESCO. (2019). La UNESCO alerta sobre la necesidad de mayor presencia de conceptos como el conocimiento del mundo, el cambio climático y la equidad de género en los currículos de América Latina y el Caribe. Oficina Regional de Educación Para America Latina y El Caribe. https://es.unesco.org/news/resultados-analisiscurricular 
Vega-Malagón, G., Ávila-Morales, J., Vega-Malagón, A. J., Camacho-Calderón, N., Becerril-Santos, A., \& Leo-Amador, G. E. (2014). Paradigmas en la investigación. Enfoque cuantitativo y cualitativo. European Scientific Journal, 10(15).

Wechsler, S. M., Saiz, C., Rivas, S. F., Vendramini, C. M. M., Almeida, L. S., Mundim, M. C., \& Franco, A. (2018). Creative and critical thinking: Independent or overlapping components. Thinking Skills and Creativity, 27, 114-122. 